\title{
BMJ Longitudinal cohort protocol study of open oropharyngeal dysphagia: relationships to gross motor attainment, growth and nutritional status in preschool children with cerebral palsy
}

To cite: Benfer KA, Weir KA, Bell $\mathrm{KL}$, et al. Longitudinal cohort protocol study of oropharyngeal dysphagia: relationships to gross motor attainment, growth and nutritional status in preschool children with cerebral palsy. BMJ Open 2012;0:e001460. doi:10.1136/bmjopen-2012001460

- Prepublication history and additional material for this paper are available online. To view these files please visit the journal online (http:// dx.doi.org/10.1136/bmjopen2012-001460).

Received 11 May 2012 Accepted 29 June 2012

This final article is available for use under the terms of the Creative Commons Attribution Non-Commercial 2.0 Licence; see http://bmjopen.bmj.com

For numbered affiliations see end of article

Correspondence to: Katherine Benfer; katherine. benfer@uqconnect.edu.au

\section{ABSTRACT}

Introduction: The prevalence of oropharyngeal dysphagia (OPD) in children with cerebral palsy (CP) is estimated to be between $19 \%$ and $99 \%$. OPD can impact on children's growth, nutrition and overall health. Despite the growing recognition of the extent and significance of health issues relating to OPD in children with $\mathrm{CP}$, lack of knowledge of its profile in this subpopulation remains. This study aims to investigate the relationship between $\mathrm{OPD}$, attainment of gross motor skills, growth and nutritional status in young children with $\mathrm{CP}$ at and between two crucial age points, 18-24 and 36 months, corrected age.

Methods and analysis: This prospective longitudinal population-based study aims to recruit a total of 200 children with CP born in Queensland, Australia between 1 September 2006 and 31 December 2009 (60 per birth-year). Outcomes include clinically assessed OPD (Schedule for Oral Motor Assessment, Dysphagia Disorders Survey, Pre-Speech Assessment Scale, signs suggestive of pharyngeal phase impairment, Thomas-Stonell and Greenberg Saliva Severity Scale), parent-reported OPD on a feeding questionnaire, gross motor skills (Gross Motor Function Measure, Gross Motor Function Classification System and motor type), growth and nutritional status (linear growth and body composition) and dietary intake (3 day food record). The strength of relationship between outcome and exposure variables will be analysed using regression modelling with ORs and relative risk ratios.

Ethics and dissemination: This protocol describes a study that provides the first large population-based study of OPD in a representative sample of preschool children with $\mathrm{CP}$, using direct clinical assessment. Ethics has been obtained through the University of Queensland Medical Research Ethics Committee, the Children's Health Services District Ethics Committee, and at other regional and organisational ethics committees. Results are planned to be disseminated in six papers submitted to peer reviewed journals, and presentations at relevant international conferences.

\section{INTRODUCTION}

Children with cerebral palsy (CP) may have poor feeding skills, influencing their growth, nutrition and overall health. ${ }^{12} \mathrm{CP}$ is the most common cause of physical disability in childhood, estimated at 2 per 1000 live born infants within Australia. ${ }^{3} \mathrm{CP}$ is an umbrella term which describes a group of disorders of movement and/or posture and motor function, which is permanent but not unchanging and due to a non-progressive interference/ lesion in the developing brain. ${ }^{4}$ Individuals with $\mathrm{CP}$ are a heterogeneous group, varying by severity and extent of motor involvement, type of movement patterns, aetiology and related conditions. ${ }^{3}$

The neurological lesion associated with $\mathrm{CP}$ may impact on the muscles of the jaw, cheeks, lips, tongue, palate and pharynx ${ }^{5}$ which manifest functionally as difficulties with controlling saliva, eating, drinking, swallowing and speaking. Eating and drinking are complex sensorimotor activities, which can be described in four phases, including the oralpreparatory, oral (propulsive), pharyngeal and oesophageal phases of the swallow. ${ }^{6}$ This study will focus on oropharyngeal dysphagia (OPD) in young children with CP, defined as impairment to any component of the oral and/or pharyngeal phases associated with eating, drinking or controlling saliva.

The oral-preparatory phase is initiated when food/fluid is taken into the mouth, and involves tasks necessary in bolus formation, including sucking, munching and chewing. Food and fluid are contained in the oral cavity surrounded by the upper dental arch and closure of the lips. Posterior leakage of the fluid bolus is prevented by contact between the soft palate and tongue; 
however, this contact is not maintained during the processing of the solid food bolus. The oral (propulsive) phase involves the backward propulsion of the food bolus, by the tongue gradually expanding its contact with the hard palate posteriorly, to initiate the pharyngeal swallow. ${ }^{6}$ The duration and movements necessary for the oral phases differ depending on the child's age and the utensils used to ingest food/fluid. ${ }^{7}$ The oralpreparatory phase of the swallow also differs when ingesting food compared to fluid boluses. When defining the swallow stages for solid foods, Matsuo and Palmer $^{6}$ advocate the use of the Process Model of Feeding, because of the overlap between the phases described in the Four Stage/Phase Model for fluids. The Process Model divides the oral-preparatory phase into Stage I Transport and Food Processing, in which the food is first ingested and moved onto the lateral occlusal surfaces of the teeth before being masticated to an optimal consistency for swallowing.

The pharyngeal phase is used to describe the passage of both food and fluid boluses through the pharynx, although when ingesting fluids it normally overlaps with the oral propulsive phase. ${ }^{6}$ On initiation of the pharyngeal phase, the soft palate elevates to seal the nasopharynx to prevent nasal regurgitation. The tongue base retracts, propelling the bolus posteriorly against the pharyngeal walls followed by the pharyngeal constrictor muscles contracting to squeeze the bolus downward. To ensure airway safety during bolus passage, respiration ceases momentarily, the vocal folds close, the arytenoids tilt forward to contact the base of the epiglottis, the larynx elevates under the base of the tongue and the epiglottis tilts backward to seal the laryngeal vestibule. The opening of the upper oesophageal sphincter (UOS) is facilitated through the relaxation of the cricopharyngeous muscle, contraction of the suprahyoid and thyrohyoid muscles, and the pressure of the descending bolus. ${ }^{6}$ The oesophageal phase is the final phase of the swallow, which begins as the bolus moves through the UOS, to be transported via automatic peristaltic waves to the stomach. ${ }^{7}$

Specific patterns of oral, pharyngeal and oesophageal impairments in feeding have been documented in children with CP. They may have difficulty in the oral phase of the swallow due to inadequate function of the oral muscles, exaggerated oral reflexes and altered oral sensitivity. ${ }^{8}$ This may include limitations to tongue lateralisation necessary for chewing solids, excessive tongue thrusting, impaired bolus transit, increased oral transit time (greater than 3s) and reduced ability to clear food residue in the mouth. Poor control of the lips may result in difficulty receiving the bolus (eg, sipping from a cup or clearing a spoon), difficulty sucking from a bottle or straw, anterior loss of food due to poor lip seal and excessive saliva loss. ${ }^{8}$ Children may also have pharyngeal phase impairments, including delayed or incomplete closure of the airway during the swallow, oropharyngeal aspiration of food or fluid and food residue in the pharynx. ${ }^{9}$ Aspiration is defined as passage of material below the vocal folds. ${ }^{6}$ This can be oropharyngeal aspiration (primary) of orally ingested material, saliva or mucous secretions; or reflux aspiration (secondary) of gastro-oesophageal refluxate. Aspiration can occur before the swallow (due to lingual disco-ordination allowing the bolus to prematurely spill over the base of the tongue, or a delayed swallow trigger); during the swallow (associated with ineffective laryngeal closure or disco-ordination); or after the swallow (related to laryngeal/pharyngeal residue falling into the reopened airway). ${ }^{6}$ Usually food entering the laryngeal vestibule and subglottic space triggers a cough, which is a major protective mechanism of the airway. ${ }^{6}$ Silent aspiration occurs when food or fluid enters below the true vocal folds with the absence of clinical signs or symptoms, which is commonly reported in children with $\mathrm{CP}^{9}{ }^{10}$ Gastrointestinal impairments (including reduced motility and reflux) occur frequently in individuals with feeding problems and CP, both secondary to and contributing towards the difficulty. ${ }^{11}$

It is believed that OPD is highly prevalent in individuals with CP; however, there is a lack of comprehensive population-based data. ${ }^{5}{ }^{12-26}$ Estimates of prevalence vary significantly, from $19 \%$ in a large register sample, ${ }^{24}$ to $99 \%$ in a sample of children with moderate-severe gross motor impairment. ${ }^{14}$ Much of the literature exploring OPD in feeding has been limited by study methodology and case-definition of OPD. Many studies have based the prevalence of OPD on parent report or non-validated methods, and samples have generally been limited to individuals with more severe gross motor impairments $^{12} \quad 14 \quad 15 \quad 17$ and across a broad age range. $^{5} 12$ 14-22 26 The findings from key studies have been summarised in table 1 .

Indirect or inconsistent means of OPD case identification have regularly been utilised in studies, with OPD identified through parent report, ${ }^{12}{ }^{13}{ }^{15-17}$ chart reviews $^{5} 17$ and non-standardised assessments. ${ }^{21-23} 25$ The variability in the method of case identification limits comparisons between these studies, and makes it difficult to estimate the true prevalence of OPD in the paediatric CP population. Parents have been shown to underestimate the presence of impaired feeding skills compared to formal clinical evaluation, ${ }^{14}$ so prevalence data using these methods may represent an underestimate of the true population prevalence of OPD. Most parent questionnaires in the reported studies lacked adequate validity and reliability data, reducing confidence in these results. ${ }^{12} 1315-17$

The generalisability of prevalence estimates of OPD to the general population of children with $\mathrm{CP}$ has been limited in most studies due to a focus on feeding skills in children with moderate-severe gross motor impairment. $^{12} \quad 14 \quad 15 \quad 17 \quad 25$ Many of the studies which sampled across the range of gross motor severity have still had a disproportionate number of individuals from the more severe classifications. ${ }^{5} 16182021$ This is largely due to 
Table 1 Prevalence of oropharyngeal dysphagia in children with cerebral palsy and its relationship to gross motor function

\begin{tabular}{ll}
\hline $\begin{array}{l}\text { Author and } \\
\text { year }\end{array}$ & Participants \\
\hline $\begin{array}{l}\text { Santoro et al } \\
\text { (2012) }\end{array}$ & $\begin{array}{l}\mathrm{n}=40 \text { children with } \mathrm{CP} \text { and } \\
\text { feeding problems aged } \\
4 \text { months }-11 \text { years, }\end{array}$ \\
& $\begin{array}{l}\text { GMFCS III-V } \\
\mathrm{n}=120 \text { children with CP, }\end{array}$ \\
$\begin{array}{ll}\text { Erkin et al } \\
(2010)\end{array}$ & $2-18$ years
\end{tabular}

OPD measure

Parent questionnaire and mealtime observation by SP

Informal observations of feeding behaviours

Cl
two groups)

CP motor type

Question on standardised assessment for register ('absent' or 'present') median $5 ; 11$ years, GMFCS I-V

(2010)

$\mathrm{n}=37$ children with $\mathrm{CP}$,

Wilson and Hustad (2009) 11-58 months (mean 41 months)

Parent report on feeding and swallowing

measure

GMFCS

CP motor type

GMFCS

CP motor type Europe Project)

No analysis of motor severity
Clinical evaluation of

OPD (no formal tools)

$\begin{array}{ll}\begin{array}{l}\text { Ortega et al } \\ \text { (2009) }\end{array} & \mathrm{n}=53 \text { children with CP, } \\ & 3-13 \text { years, GMFCS I-V } \\ & \text { (with } 75 \% \text { of sample from } \\ & \text { IV-V) } \\ \text { Calis et al } & n=166 \text { children with } \\ \text { (2008) } & \text { severe CP and ID, } \\ & 2-19 \text { years (mean } \\ & 9 ; 4 \text { years). GMFCS IV-V, } \\ & \text { IQ }<55\end{array}$

Yilmaz, et al (2004)

Field et al (2003)

Fung et al (2002)

$\mathrm{n}=23$ children with spastic I-V

$\mathrm{n}=44$ children with $\mathrm{CP}$, 1 month-12 years (median age range 13-36 months) $\mathrm{n}=230$ children with $\mathrm{CP}$, 2-18 years (mean 9.7 years), GMFCS III-V

Sullivan et al (2000)

Scale

DDS and DSS

Parent report

FFAm

Record review

Parent reported on feeding questionnaireand severe

Register question to determine 'articulation/ $\mathrm{n}=271$ parents of children with childhood impairments (96\% CP), 4-13 years, mild-severe gross motor
GMFCS (collapsed to

Questionnaire

\section{Gross motor}

(Surveillance of CP in

Oral Motor Assessment GMFCS

GMFCS

Ambulatory status

No analysis of motor severity

GMFCS rated as none, mild, mod swallowing problems' Parent questionnaire to

Parent rated severity of motor function, relating to aids needed (mild, mod
Major findings

Children from GMFCS III showed best feeding performance (hemi/ diplegic $\mathrm{CP}$ )

$22 \%$ feeding dysfunction (12\% mild, $8 \%$ moderate and $2 \%$ severe)

Feeding dysfunction in 4\% of GMFCS I-III, and 22\% of GMFCS IV-V $(p<0.001)$ $19 \%$ chewing and swallowing problems

GMFCS significantly related to swallowing/ chewing difficulties and excessive drooling:

GMFCS IV-OR 4.8 GMFCS V-OR 15.7

$56 \%$ had difficulty feeding from a bottle

$78 \%$ had oral motor involvement (including motor speech)

No analysis with gross motor

$83 \%$ did not have functional feeding skills No analysis with gross motor 99\% clinically apparent dysphagia Oral motor severity positively associated with motor functional severity $(p<0.001)$

Postural stability positive association to DDS score, but not postural alignment for eating 50-74\% normal-mild feeding difficulties; $30-51 \%$ moderate-severe feeding difficulties $68 \%$ oral motor delay $32 \%$ dysphagia

$48 \%$ feeding problems GMFCS level was highly associated with the degree of feeding dysfunction $(p<0.001)$ and severe) 
Table 1 Continued

\begin{tabular}{|c|c|c|c|c|}
\hline $\begin{array}{l}\text { Author and } \\
\text { year }\end{array}$ & Participants & OPD measure & $\begin{array}{l}\text { Gross motor } \\
\text { measure }\end{array}$ & Major findings \\
\hline & & $\begin{array}{l}\text { investigate specific } \\
\text { feeding problems }\end{array}$ & & $\begin{array}{l}\text { range of specific feeding } \\
\text { problems }(e g, \text { choking with } \\
\text { food } p<0.001 \text {; prolonged } \\
\text { mealtime } p<0.001)\end{array}$ \\
\hline $\begin{array}{l}\text { Reilly et al } \\
\text { (1996) }\end{array}$ & $\begin{array}{l}\mathrm{n}=49 \text { children with } \mathrm{CP} \text {, } \\
12-72 \text { months, } \\
\text { mild-profound ( } 70 \% \text { with } \\
\text { severe-profound } \mathrm{imp} \text { ) }\end{array}$ & $\begin{array}{l}\text { SOMA } \\
\text { Early feeding histories }\end{array}$ & $\begin{array}{l}\text { Standard Recording } \\
\text { of Central Motor } \\
\text { Deficit-classified as } \\
\text { no disorder/mild; } \\
\text { severe/profound }\end{array}$ & $\begin{array}{l}\text { Positive relationship } \\
\text { between OPD severity and } \\
\text { gross motor severity } \\
(p=0.000) \\
\text { Mod and severe OPD } \\
\text { more common in } \\
\text { tetraplegia, whereas } \\
\text { diplegia was associated } \\
\text { with mild OPD }(p=0.001)\end{array}$ \\
\hline $\begin{array}{l}\text { Dahl et al } \\
\text { (1996) }\end{array}$ & $\begin{array}{l}\mathrm{n}=35 \text { children with } \mathrm{CP} \text {, } \\
2.4-15.2 \text { years (mean } \\
7.7 \text { years), profound motor } \\
\text { handicaps (moderate and } \\
\text { severe CP) }\end{array}$ & $\begin{array}{l}\text { Parent interview } \\
\text { (retrospective data of } \\
4 \text { weeks) triangulated } \\
\text { with medical file review }\end{array}$ & $\begin{array}{l}\text { Motor severity } \\
\text { differentiated by level } \\
\text { of dependence }\end{array}$ & $\begin{array}{l}60 \% \text { reported as having } \\
\text { daily feeding problems } \\
\text { No analysis of gross motor }\end{array}$ \\
\hline $\begin{array}{l}\text { Stallings et al } \\
\text { (1993) }\end{array}$ & $\begin{array}{l}n=142 \text { children with } \\
\text { quadriplegic } C P \text {, } \\
2-18 \text { years }\end{array}$ & $\begin{array}{l}\text { Parent interview }(0-5 ; \\
0=\text { no problems, } 5=\text { all }(5) \\
\text { oral motor problems) }\end{array}$ & $\begin{array}{l}\text { Diagnostic criteria } \\
\text { (for quadriplegic CP) } \\
\text { not defined in paper }\end{array}$ & $\begin{array}{l}86 \% \text { impaired oral motor } \\
\text { ability } \\
\text { No analysis of gross motor }\end{array}$ \\
\hline $\begin{array}{l}\text { Waterman et al } \\
\text { (1992) }\end{array}$ & $\begin{array}{l}n=56 \text { children with } C P \text {, } \\
5-21 \text { years (median } \\
14 \text { years), mild-severe }\end{array}$ & $\begin{array}{l}\text { Chart review (clinical or } \\
\text { radiographical dysphagia) } \\
\text { Interviews with SP }\end{array}$ & $\begin{array}{l}\text { Severity defined } \\
\text { based on ambulatory } \\
\text { status from chart } \\
\text { review }\end{array}$ & $\begin{array}{l}27 \% \text { had evidence of } \\
\text { swallowing disorders } \\
\text { More severe CP in } \\
\text { dysphagic group } \\
\text { ('consistent but } \\
\text { non-significant trend'-no } \\
\text { statistics reported) }\end{array}$ \\
\hline $\begin{array}{l}\text { Thommessen } \\
\text { et al (1991) }\end{array}$ & $\begin{array}{l}n=42 \text { children with } C P \text {, } \\
1-16 \text { years }\end{array}$ & $\begin{array}{l}\text { OPD evaluated by } 3 \text { OTs/ } \\
\text { PTs (based on child's } \\
\text { age) }\end{array}$ & $\begin{array}{l}\text { No analysis of motor } \\
\text { severity }\end{array}$ & $\begin{array}{l}33 \% \text { had OPD } \\
\text { No analysis of gross motor }\end{array}$ \\
\hline $\begin{array}{l}\text { Love et al } \\
\text { (1980) }\end{array}$ & $\begin{array}{l}n=60 \text { children with } C P \text {, } \\
3-23 \text { years (mean } \\
12.5 \text { years), spastic, } \\
\text { athetoid and mixed; } \\
\text { mild-non-ambulatory }\end{array}$ & $\begin{array}{l}\text { Non-standardised } \\
\text { oral-motor tasks (biting, } \\
\text { sucking, swallowing, } \\
\text { chewing soft and firm } \\
\text { food) }\end{array}$ & $\begin{array}{l}\text { No analysis of motor } \\
\text { severity }\end{array}$ & $\begin{array}{l}40 \% \text { with inadequate } \\
\text { feeding }\end{array}$ \\
\hline
\end{tabular}

sampling bias, with most studies recruiting from special schools or clinic databases, thus limiting the sample representativeness. In addition, a range of measures have been used to determine gross motor severity, including formal classification systems such as the Gross Motor Function Classification System (GMFCS), and criteria developed for the individual study. This limits our ability to accurately quantify the prevalence of OPD across the full range of gross motor severity, from mild to severe, and may provide an overestimate of the prevalence in the general population of children with CP if rates are extrapolated based on the moderate-severe sample.

Feeding skills develop rapidly in the early years as children transition through a range of food and fluid textures, related to their developing anatomy, neurology and physiology. ${ }^{27}$ Rapid development of sensorimotor integration of swallowing and respiration, upper limb skills, posture and psychosocial maturation occur during the first 3 years. $^{7}$ By 18 months children are typically sitting independently, with fully co-ordinated swallow and respiration, and taking a full range of textures. ${ }^{7}$ The development of chewing skills continues into childhood, with the adult co-ordination of lateral and vertical jaw movements emerging between three and 6 years. ${ }^{28}$ Most prevalence studies of OPD in children with CP have been designed to examine oral sensorimotor skills in samples with a broad age range from early childhood (4 months to 4 years) through to adolescence or early adulthood (11-25 years)..$^{5} 12$ 14-19 2126 The mean age for many of these studies was 9 years. Only two studies 
limited their sample to preschool years, with participants ranging in age from 12 to 72 months $^{20}$ and 11 to 52 months. ${ }^{13}$ Few children from the toddler or preschool age range have been sampled in previous studies, so a gap in knowledge remains. It is important to begin to delineate OPD in this critical age range to facilitate early identification and intervention, and to explore the progression of early feeding skills and their changing relationships with other associated factors (eg, growth, nutrition and respiratory health).

It is well accepted clinically that there is an interaction between an individual's oral sensorimotor skills in feeding and their gross motor skills. An individual's feeding posture can impact on their swallow by promoting poor alignment or reducing the stability for controlled oral movements, as well as the influence of the neurological lesion on all motor skills. ${ }^{15} 29$ Poor head position has been related to compromised airway protection by opening the airway, and influencing the flow rate of foods/fluids swallowed. ${ }^{30}$ The precise relationship between body position and swallow-breath coordination continues to be explored. ${ }^{31}$ This relationship between OPD and gross motor skills is supported in the literature, with the prevalence and severity of OPD reported to be positively correlated with the extent of motor involvement. ${ }^{5}$ 14-16 2024 However, these findings lack weight due to few studies using direct objective measures of oral sensorimotor skills, ${ }^{5}{ }^{14-16} 2324$ a lack of validated measures of gross motor skills ${ }^{5} 16$ or sampling only children with moderate-severe gross motor impairment. ${ }^{14} 1525$

The Oxford Feeding Study of 271 children with OPD, found those with more extensive motor involvement, that is, quadriplegia and dyskinesia, were most likely to have difficulties with swallowing and articulation, based on parent report. ${ }^{16}$ Those unable to walk or who required an aid and helper to walk were more likely to have problems eating and swallowing lumpy food, to need food mashed or liquidised, and were also more likely to be fed via a tube. In a large register-based study ( $n=1357$ ), the odds of having swallowing/chewing difficulties and excessive drooling increased significantly as GMFCS level increased; ${ }^{24}$ however, this study only used a single standardised question to determine the presence of feeding difficulty. Using validated assessments (Schedule for Oral Motor Assessment (SOMA) and Standard Recording of Central Motor Deficit categories), the presence of gross motor impairment was significantly associated with the presence of oral motor dysfunction in a cross-sectional community-based sample of 49 preschool children with CP. $^{20}$ While strengthened by using validated measures for both oral motor and gross motor skills, the sample was small and only used binary outcomes (presence/absence of dysfunction). The relationship between OPD and gross motor skill attainment will be strengthened by exploring this association across a number of gross motor severity levels using the GMFCS.
The feeding impairments resulting from OPD may impact negatively on many dimensions of an individual's health, including the child's development, growth and nutrition, chest status and respiratory health, gastrointestinal functioning and parent-child interactions. ${ }^{32}$ Both OPD and tube feeding are demonstrated risk factors for increased premature mortality in individuals with CP. ${ }^{33-35}$ Optimal nutrition in the early years forms a critical foundation for improved health across the lifespan. Compromised nutritional status influences children's mood and irritability, muscle spasticity, healing, peripheral circulation and general well-being. ${ }^{36}$ In addition, OPD can result in acute and/or chronic oropharyngeal aspiration which is significantly associated with compromised respiratory status, including recurrent lower respiratory tract infections and chronic lung disease. ${ }^{9}{ }^{19}$ Understanding the nature and severity of OPD in young children with CP and its relationship to gross motor attainment, growth and nutritional status, will inform health interventions, benefiting children with $\mathrm{CP}$ and their families, and potentially lowering costs of healthcare. $^{37}$

\section{Aims and hypotheses}

This study will investigate the relationship between OPD, gross motor skills, growth and nutritional status in young children with CP across two critical age points, 18-24 and 36 months, corrected age. Specifically, this study aims to:

1.

A. Systematically review the literature determining the clinimetrics of measures of OPD in preschool children with CP.

B. Test the psychometric properties of the SOMA, Dysphagia Disorders Schedule (DDS) and Pre-Speech Assessment Scale (PSAS) in young children with CP.

2.

A. Determine the prevalence of OPD and its subtypes (impaired saliva control, oral phase impairment and pharyngeal phase impairment) in a population of children with $\mathrm{CP}$ at $18-36$ months.

B. Explore the nature of the relationship between OPD and gross motor functional severity (according to GMFCS levels); and growth and nutritional status.

3. Longitudinally examine the potential risk factors for OPD (including gross motor attainment, anthropometric measures, dietary intake, ingestion functions, food and fluid textures, gender, age and socioeconomic factors) in children aged 18-24 and 36 months with CP.

These aims will be explored through the following three hypotheses:

$\mathrm{H}_{1}$ : The SOMA and DDS will be the most valid and reliable measures of OPD in young children with CP. The PSAS will have the best clinical utility. 
$\mathrm{H}_{2}$ :

A. There will be a negative relationship between OPD prevalence and gross motor function in children with CP aged 18-36 months.

B. There will be a positive relationship between OPD prevalence, poor growth and nutritional status in children with CP aged 18-36 months.

$\mathrm{H}_{3}$ : Gross motor function, poor growth and nutritional status will have a greater association with OPD in children with CP than demographic risk factors.

\section{Study significance}

The results of this study will:

- Determine the accuracy of the SOMA, DDS, PSAS and signs suggestive of pharyngeal phase impairment, in detecting and evaluating OPD in preschool-aged children with CP.

- Contribute population-based data on the prevalence of OPD and subtypes, in children with CP using standardised measures. To date there is limited comprehensive population data across all gross motor severity levels. These data are essential before intervention trials can be conducted.

- Delineate the relationship between OPD and gross motor skill attainment in children with CP. Greater understanding of this relationship will assist in proactive screening in early intervention services, including early detection of children at risk of aspiration and compromised chest status, and prevention of negative health effects.

- Further explore potential associations between OPD and nutritional status and growth in children with CP. This will allow for greater access to preventative nutritional treatments and the development of more targeted interventions, thus promoting growth and overall health outcomes in young children with CP.

\section{METHODS AND ANALYSES}

This prospective longitudinal cohort study aims to recruit 200 children with CP born in Queensland, Australia, between 1 September 2006 and 31 December 2009. The OPD study is part of a larger longitudinal population-based study, Queensland CP Child: Growth, Nutrition and Physical Activity, which is exploring growth, nutrition and physical activity in children with CP (National Health and Medical Research Council (NHMRC) Australia, 569605). This study is being conducted in conjunction with another study, Queensland CP Child: Motor Function and Brain Development Study (NHMRC 465128). Figure 1 visually represents the relationship between these studies and the OPD substudies, which include:

1. Validity and reproducibility studies

A. Discriminative validity with typically developing reference sample;

B. Convergent validity with an additional OPD measure;
C. Reproducibility (test-retest, intrarater, inter-rater).

2. Cross-sectional study of children aged 18-36 months

A. Overall prevalence of OPD, subtypes and association with gross motor;

B. Oral phase impairment;

C. Pharyngeal phase impairment;

D. Functional feeding skills on food and fluid textures.

3. Longitudinal study of children between $18-24$ and 36 months.

\section{Recruitment}

State-wide subject recruitment started in April 2009 in collaboration with the Queensland Cerebral Palsy Register, the Queensland Cerebral Palsy League, the Royal Children's Hospital (RCH) Brisbane, the Queensland Cerebral Palsy Health Service, the Royal Women's Hospital Brisbane and the Mater Children's Hospital. Paediatricians, general practitioners, allied health professionals, child health nurses and neonatal follow-up clinics are encouraged to refer children with motor delay (not sitting at 10 months, not standing at 12 months or walking at 24 months) for confirmation of a diagnosis of $\mathrm{CP}$ at the $\mathrm{RCH} /$ Mater Mothers' Hospital Specialist clinics. High ascertainment is expected for children across all levels of motor severity (GMFCS I-V) particularly as many of these children access services through the Queensland Cerebral Palsy Health Service, one of the key referral sources. Children who are detected after 18 months of age will be entered into the study later, at the time of diagnosis. Children can enter the study at 18, 24, 30 or 36 months age points. Those entering at 18 or 24 months will have their second assessment point collected at 36 months, and will be included as part of the longitudinal study. Children entering at 30 or 36 months will have their second assessment at 48 months, and therefore will not be included in the longitudinal study detailed in this study protocol. Further details of study entry and feasibility can be found in the larger study's protocol. ${ }^{38}$

Forty children with typical development aged 1836 months (stratified for age) will be recruited to participate as a reference sample for the study. Siblings of children participating in the overall study will be invited to participate, as well as recruitment through staff newsletters, a hospital childcare centre and participants from other studies within the centre.

\section{Selection criteria}

Inclusion criteria

Children aged 18-36 months corrected age at the time of evaluation (birth-years 2006-2009), born in Queensland, with a confirmed diagnosis of $\mathrm{CP}$ are invited to participate in the present study. For the present study, CP is defined as a disorder of movement and/or posture and motor function, which must be permanent but not unchanging, and due to a non-progressive 


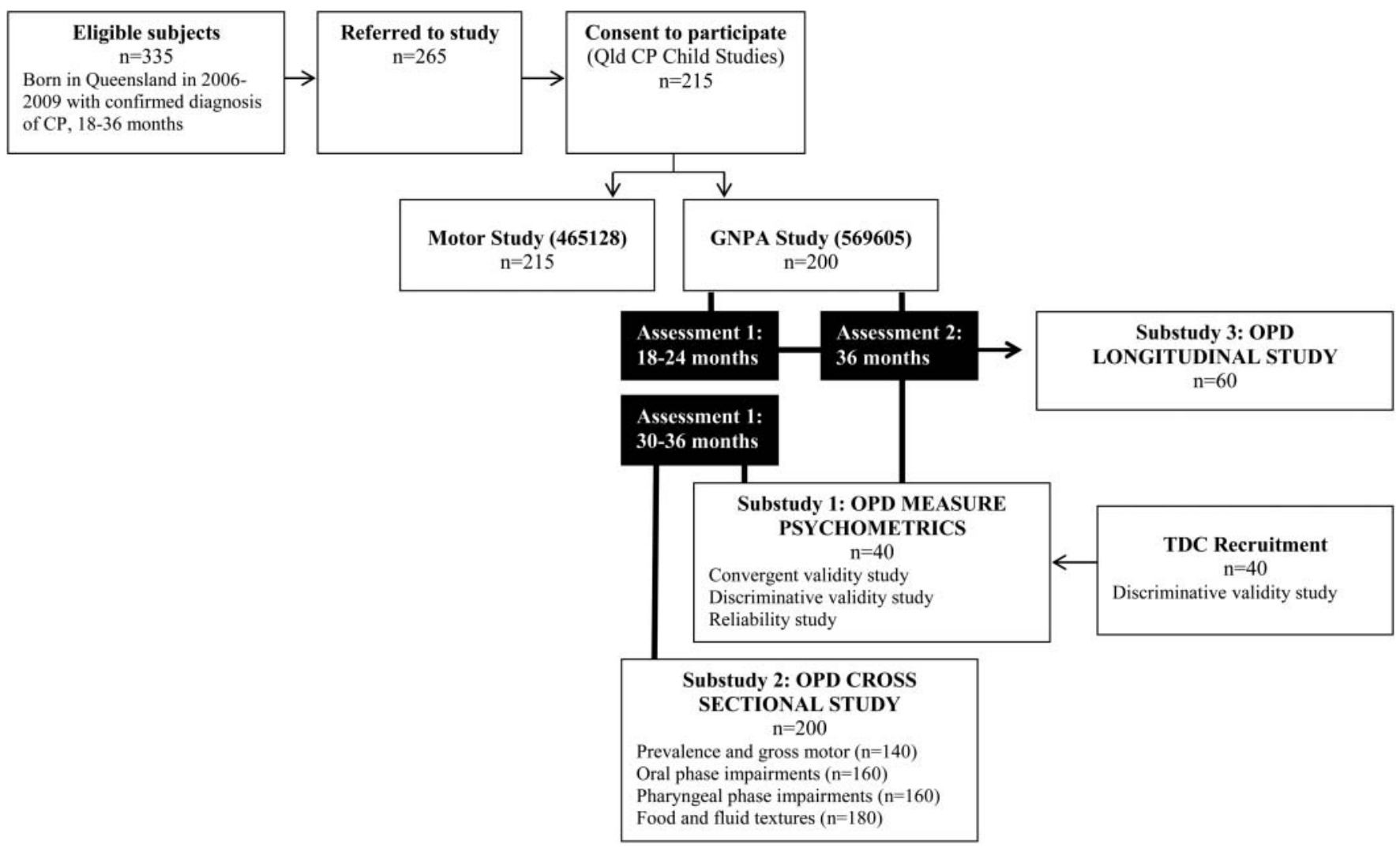

Figure 1 Critical pathways for oropharygeal dysphagia study. CP, cerebral palsy; GPNA, growth nutrition and physical activity; OPD, oropharyngeal dysphagia; Qld, Queensland; TDC, typically developing children.

interference/lesion in the developing brain (congenital lesions only). ${ }^{4}$ The characteristic motor types are spasticity and dyskinesias (ataxia, rigidity and dystonia), and clinical features may also include negative signs of the motor neurone syndrome (muscle weakness and poor selective motor control) ${ }^{39}$

\section{Exclusion criteria}

Children diagnosed with a progressive or neurodegenerative lesion and children born outside Queensland are excluded from the study.

\section{Typically developing reference sample}

Children are eligible to participate in the reference sample if they are aged 18-36 months; born full term ( $<37$ weeks); with no admissions to neonatal care, no diagnosis receiving medical or allied healthcare; and not on regular medications.

\section{Measurements and procedures}

Following confirmation of a diagnosis of CP, children attend the RCH for an assessment session with their family. During this visit, children are assessed using the Gross Motor Function Measure (GMFM), ${ }^{40}$ Manual Ability Classification System (MACs), ${ }^{41}$ anthropometric measurements taken, questionnaires administered to the parent/caregiver verbally (including the Pediatric Evaluation of Disability Inventory, ${ }^{42}$ and Queensland CP
Child: Growth, Nutrition and Physical Activity: Feeding Questionnaire) and the child's mealtime is videotaped.

Children participating in the reproducibility study will be invited to return to the hospital within a month to have a repeat mealtime video. If this is not possible, a home visit will be conducted. Children participating in the typically developing reference sample will be assessed at the hospital or at home, for a single mealtime video.

\section{Feeding evaluation}

During the feeding assessment, the child is well positioned in their typical mealtime seating (ie, chair, stroller and carer's arms). The video camera is set up to include a view of the child's face and neck, angled to the side of the feeder's shoulder of the hand that is not feeding the child, as per the study snack protocol. Prior to and following the mealtime, the researcher videoing the session records observations regarding clinical swallow signs (wet/gurgly voice, wet/gurgly breathing, rattly chest or the presence of cough) and severity of drooling. These ratings are confirmed by the speech pathologist when rating the videos. During the video session, the child is given three standardised presentations of each of four textures (puree, lumpy, chewable and fluid) by their primary carer, as outlined in the SOMA administration manual. ${ }^{43}$ Purees include foods such as yoghurt, mousse or pureed fruit. Lumpy foods 
could include semisolid (eg, baked beans, roughly mashed vegetables) or solid foods (eg, fruit salad) from a spoon. For the purpose of this assessment, chewable foods are items that are finger fed, usually requiring biting, including biscuits or whole fruit. Following these standard presentations, the child is allowed to complete the snack eating independently or assisted by their primary carer.

\section{Primary measures}

A major limitation in studies of OPD is the lack of widely accepted, validated and reliable measures. ${ }^{15}$ The aim of the present study is to gather information regarding OPD that reflects children's performance in naturalistic environments (eg, home and childcare centres). For this reason, non-invasive observational methods were selected as part of the standard protocol for all children. The SOMA, ${ }^{44}$ Dysphagia Disorders Survey: Pediatric (DDS), ${ }^{45}$ and PSAS $^{46}$ were selected through systematic review as the most appropriate non-invasive objective clinical measures for the detection of dysphagia for this study. ${ }^{47}$ The video tapes of children's mealtimes are formally rated by an independent speech pathologist, and data recorded using the standard assessment forms. Sixteen clinical pharyngeal signs suggestive of aspiration are also rated for each food/fluid texture, in conjunction with the rating completed in the session. The use of videos in mealtime observations is recommended in the SOMA administration manual to allow repeated viewing for more accurate description of motor tasks. The speech pathologist is certified in the use of the DDS to meet the validation standards. ${ }^{48}$ The allocation of GMFCS level is masked to the speech pathologist when rating mealtime videos. If clinically indicated, some children have further evaluation of their OPD using instrumental assessments, such as Video Fluoroscopic Swallow Study (VFSS). This information is collected when available but is not part of the standard protocol for all children.

\section{Schedule for Oral Motor Assessment}

The SOMA is a standardised discriminative assessment which quantifies OPD in children aged between 8 and 24 months. ${ }^{43}$ It was originally designed to evaluate children with no/mild neurological dysfunction, but subsequently was used to evaluate oral motor dysfunction (OMD) associated with a number of causes including neurological impairments. ${ }^{43}$ The tool categorises children as OMD or normal oral motor function based on specified thresholds for each of seven oral motor challenge categories (OMCC) (puree, semi-solid, solid, cracker, bottle, trainer cup and cup). ${ }^{43}$ The tool is predominantly a test of oral phase dysfunction; however, some items pertain to swallowing and the pharyngeal phase. Children are only scored on food/ fluid textures they accept during the assessment. The standardised administration of textures outlined in the administration manual is maintained in this study as much as possible, while allowing some flexibility for individual child and family factors to optimise the naturalistic context of the assessment.

The SOMA has been validated on 127 young infants; 58 comparison children with typical oral skills, 56 with non-organic failure to thrive (aged 8-24 months), and 13 children with CP and overt feeding difficulties (aged up to 42 months). ${ }^{49}$ The abnormality score (total number of OMCCs with OMD) for children with CP was significantly different from the comparison group $(p<0.0001)$. Individual OMCCs do not have adequate discriminative validity reported to be analysed as individual subtests, with $8-77 \%$ false negatives in the CP group. ${ }^{49}$ The reliability of the measure was established by two independent speech pathologists rating three trials of 10 randomly selected videos from the sample. It has strong inter-rater reliability ( $\kappa=1.0$ in $68 \%$ of fluid category items and $58 \%$ of food category items) and test-retest reliability between boluses ( $\kappa=1.0$ in $84 \%$ of items)..$^{50}$

\section{Dysphagia Disorders Survey}

The DDS was developed as an evaluative screening tool to assess feeding and swallowing function in children and adults with a developmental disability. ${ }^{48}$ Through observation of a typical mealtime, it identifies those with signs of oral preparation, oral initiation, pharyngeal and oesophageal phase dysphagia. ${ }^{48}$ The measure is divided into two distinct parts: Part 1 scores dysphagia-related consequences (such as low weight, adaptive utensils and position); Part 2 rates the specific oral functions observed across three textures (non-chewable food, chewable food and fluid). The raw score from Part 1, and percentiles which are derived from both Parts 1 and 2, are not used in this study as they assess consequences of mealtime difficulty rather than specifically OPD. Part 2 provides a raw score that indicates an individual's functional eating competency (with a maximum impairment raw score of 22) and has been used previously as a measure of OPD. ${ }^{14}$

The DDS underwent final standardisation on 427 individuals with mean age of 33 years. ${ }^{14}$ The paediatric measure was developed in a group of 166 children (range 2 years 1 month-19 years 1 month; mean 9 years 4 months), with moderate-severe CP (GMFCS III-V) and intellectual disability. ${ }^{14}$ Test validity and interitem reliability were derived from an initial sample of 626 people with developmental disability. ${ }^{51}$ Convergent validity was demonstrated in two studies comparing DDS scores to blinded speech pathologist diagnosis. ${ }^{14}{ }^{48}$ Inter-rater reliability of $97 \%$ agreement was calculated from a sample of 21 participants rated by six speech pathologists (each pair of speech pathologists rated seven participants). ${ }^{48}$

\section{Dysphagia Severity Scale}

The Dysphagia Severity Scale was developed by Calis et $a l^{14}$ to provide a severity rating from the DDS Part 2 raw scores. Individuals are classified as one of the four severity levels, with level one being no disorder, and level four a profound disorder. The mild classification 
and moderate-severe classification are differentiated by the presence of pharyngeal phase impairments (items 13-14 on the DDS), in addition to a score of one or more on the DDS Part 2. A profound disorder is reflected by non-oral status of individuals due to the severity of their OPD.

\section{Pre-Speech Assessment Scale}

The PSAS is an evaluative measure that examines 27 pre-speech feeding behaviour performance areas related to sucking, swallowing, biting, chewing, respirationphonation and sound play. ${ }^{46}$ It is appropriate for use with children with a neurological impairment, as well as those with typical development. Each subtest is scored on an ordinal abnormality scale (1-9) and a developmental scale (with age norms to $24+$ months), to provide a double score overall. This provides comprehensive information on both dysfunctional and delayed feeding behaviour expected up to 24+ months.

The PSAS was developed through a 3-year longitudinal study of six children, and field testing of the measure for 8 years by 215 trained clinicians who provided annual feedback on its clinical use. ${ }^{46}$ Other aspects of the measure's validity have not been tested. Reliability has been shown to be strong, although only in two studies with limited methodology. ${ }^{46}{ }^{44}$ Intra-rater reliability was $96 \%$ for 25 feeding behaviours which were scored in the six typically developing children. ${ }^{44}$ Inter-rater reliability for this same sample was similarly excellent between two raters $(92 \%) .{ }^{44}$ Inter-rater reliability was fair to good when rated from video footage, with 65$87 \%$ agreement when 75 clinicians' ratings were compared to a predetermined standard of correctness for 78 children. ${ }^{46}$

\section{Signs suggestive of pharyngeal phase impairment}

Premealtime and post-mealtime observations of the presence or absence of (1) wet/gurgly voice (2) wet/gurgly breathing, (3) rattly chest and (4) cough are rated face-to-face in the mealtime session by a trained research assistant, to assess clinical signs of pharyngeal phase difficulty. A determination of pharyngeal phase impairment is noted if a child demonstrates any one of these signs, or 1 of 16 signs rated from video by the speech pathologist. These behaviours include gagging, coughing, choking, vomiting, throat clearing, multiple swallows, wheezing, stridor, rapid or laboured breathing, gurgly voice, rattly chest, snuffly nose, eye tearing, circumoral cyanosis/duskiness and food refusal and are noted for each food and fluid texture. These signs were selected from the literature ${ }^{10} 45$ and research conducted by one of the investigators (KAW) ${ }^{52}$

A cross-sectional study of 150 children with dysphagia (mean age 16 months) compared retrospective data of pharyngeal phase impairments identified by VFSS to 11 commonly reported clinical signs and symptoms to determine their sensitivity and specificity. ${ }^{52}$ Wet voice (sensitivity 0.67 and specificity 0.92 ), wet breathing (sensitivity 0.33 and specificity 0.83 ) and cough (sensitivity 0.67 and specificity 0.53 ) were considered good clinical markers of oropharyngeal aspiration on thin fluids, but not for puree textures.

\section{Thomas-Stonell \& Greenburg Scale-saliva control}

The Thomas-Stonell \& Greenberg ${ }^{53}$ Scale is a semiquantitative assessment of drooling severity (one-point to fivepoint scale of no drooling to profuse drooling) and frequency (one-point to four-point scale of no drooling to constant drooling). A pre- and post-mealtime severity rating is recorded by trained researchers within the mealtime assessment and confirmed by the speech pathologist from video. In addition, a severity and frequency rating by the parents is collected based on observations during the previous week, and information reporting on the representativeness of this rating.

In a case-control study of 14 children with saliva loss and spastic CP aged 7-18 years (mean 11;7 years), drooling frequency and severity were reported by parents on the Thomas-Stonell \& Greenberg Scale. ${ }^{54}$ A Drooling Quotient, derived from parent scores, was compared to a more objective measure of weighing saliva loss on bibs and shown to be positively correlated (Spearman's $\mathrm{r}=0.604 \mathrm{p}<0.05) .{ }^{54}$

\section{Gross Motor Function Classification System}

The GMFCS is a five-level classification system of children's functional gross motor severity. It is based on selfinitiated movements, anti-gravity postures and motor skills expected in a typical 5-year-old children. ${ }^{55}$ Children who are independently ambulant are classified as GMFCS I or II, those requiring an assistive mobility device to walk classified as GMFCS III and those in wheeled mobility as GMFCS IV and V. Two physiotherapists, trained in the use of the GMFCS, independently observe and classify children in one of five functional categories. ${ }^{55}$

The GMFCS has internationally established validity, reliability and stability for the classification and prediction of motor function of children with $\mathrm{CP}$ aged 2-12 years. ${ }^{55-57}$ It has a high inter-rater reliability (generalisability $=0.93) .{ }^{56}$ Classification of gross motor abilities change with age, therefore separate descriptions are used for different age bands. In the current study, the $<2$ and 2-4 years descriptions are used. Lower inter-rater reliability is documented for the $<2$ years age band $(\kappa=0.55)$, as younger children's gross motor abilities are more variable, and less developmental information is available on which to base the classification ${ }^{58}$ Test-retest reliability from $<2$ to 12 years appeared to be acceptable (generalisability coefficient $=0.68$ ). The GMFCS has been correlated with a number of motor scales, as well as CP distribution and type of motor impairment. ${ }^{59}$

\section{Anthropometry}

Height or length (depending on children's ability to stand) is measured to the last completed millimetre by a portable stadiometer/length board (Shorr Productions, 
Maryland, USA). Where a direct measure of height or length is not possible, height is estimated using published equations from knee length or upper-arm length ${ }^{60}$ measured with an anthropometer (Holtain Ltd, Dyfed, UK). Weight (measured to the nearest $100 \mathrm{~g}$ using chair scales; Seca, Germany) and skin-fold thickness (tricep and subscapular skinfolds, measured in millimetres with Harpenden callipers (Holtain Ltd)) measures are taken and body mass index (BMI) calculated (as weight/height, $\mathrm{m}^{2}$ ) to assess children's nutritional status. Skin-fold measurements and BMI will be converted to z scores for analysis. ${ }^{61}$ All measures are conducted by trained investigators. Full details of anthropometric procedures are provided in the larger study protocol paper. ${ }^{38}$

\section{Dietary intake}

A 3-day-weighed food record is used to measure children's typical dietary intake. ${ }^{62}$ Parents are instructed on the standard protocol to ensure accuracy and consistency in completing the food record. Food records will be analysed for the percentage of children's diet made up of food and fluid textures. Food records are also analysed using the Foodworks dietary analysis software program (Xyris Software (Australia) Pty Ltd, Kenmore Hills, Australia) to give information regarding energy, carbohydrate, fat and protein intake.

\section{Secondary measures}

Queensland cerebral palsy child: growth, nutrition and physical activity: Feeding Questionnaire (QId CP Child Feeding Questionnaire)

The Qld CP Child Feeding Questionnaire gathers parent report on their child's oral sensorimotor and mealtime function. Parent report will be used to triangulate findings from clinical assessment to gain a more comprehensive picture of the child's skills across settings and time. It includes:

- Severity and frequency of saliva loss using the Thomas-Stonell \& Greenberg Scale (above).

- The impact of saliva on four domains, including the impact on child and family measured using a 10-point visual analogue scale.

- Types of food and fluid included in the child's diet: inclusion of textures rated for four fluid levels (thin, mildly thick, moderately thick and extremely thick) and five food textures (puree, thick puree, lumpy mashed food, chewable solids and tough chewable foods). Fluid terms align with the Australian Standardised Labels and Definitions. ${ }^{63}$

- Presence of eating or drinking problems: rated on a four-point scale from no feeding problems to severe difficulties. Severity is also rated for eating and drinking on a 10-point visual analogue scale.

- Mealtime behaviours and signs suggestive of pharyngeal phase impairment or aspiration are documented by parents against the same 16 signs and symptoms suggestive of pharyngeal phase impairment as is noted in clinical observation. Presence or absence of specific signs and symptoms were noted on each texture (thin fluid, thick fluid, puree, lumpy and finger foods).

\section{Gross Motor Function Measure}

Gross motor function is evaluated at each assessment using the GMFM (GMFM-66 and GMFM-88). ${ }^{40}$ The GMFM is an evaluative tool that covers five gross motor domains, including lying and rolling; sitting; crawling and kneeling; standing; and walking, running and jumping. The GMFM-66 is a subset of items from the GMFM-88, developed through Rasch analysis, and is shown to be valid and reliable in children with $\mathrm{CP}^{64}$ The GMFM-66 will be used to provide an overall measure of gross motor function, and the GMFM- 88 domain scores to explore specific motor skills. Scores are expressed as a percentage of the maximum score, which are skills expected of a typically developing child at 5 years. ${ }^{65}$ The GMFM is not valid for comparisons of children across different age ranges, therefore all analyses using GMFM scores are completed in 18-24 and 30-36 months age brackets. Gross motor assessment is completed by two experienced paediatric physiotherapists who have criterion rating with the study developers (RNB).

\section{Motor type and distribution}

The type of CP (spastic, dyskinetic and hypotonic) and motor distribution (hemiplegia, diplegia and quadriplegia) is classified according to the Surveillance of CP in Europe. ${ }^{66}$ This is assessed by two independent physiotherapists at each assessment.

\section{Manual Ability Classification System}

Children's manual ability is classified during performance in everyday activities according to the MACs. The MACs classifies children on a five-level scale based on how they use their hands when performing activities such as eating, dressing, playing and drawing. ${ }^{41}$ This classification was developed for children aged 4-18 years, but has been shown to have good reliability for use in children as young as 2 years. ${ }^{67}$ Children are rated by two independent physiotherapists.

\section{Sample size calculations}

Queensland cerebral palsy child: growth, nutrition and physical activity

On the basis of a reported incidence of CP of 2/1000 live births within Australia, there is an estimated 100 new cases of CP in Queensland each year. ${ }^{3}$ For sample size calculations, a population prevalence estimate of $90 \%$ was taken from the study by Reilly et al. ${ }^{20}$ In order to estimate the true prevalence of OPD in the population of children with $\mathrm{CP}$ with $95 \%$ confidence, a minimum sample of 35 participants were needed to provide sufficient precision within $\pm 10 \%$ of the true value.

Owing to the limited data reported in the literature of prevalence based on direct clinical evaluation in the mild gross motor level, children in GMFCS I were hypothesised to have normal feeding skills. Nearly all 
Table 2 Summary of primary outcome and exposure variables in the present study by objective and statistical tests

\begin{tabular}{|c|c|c|c|}
\hline Hypothesis & Outcome variable & Exposure variable & Statistics \\
\hline $\mathrm{H} 2(\mathrm{~A})$ & $\begin{array}{l}\text { OPD overall (yes on SOMA, DDS, PSAS } \\
\text { or clinical pharyngeal signs) Dichotomous }\end{array}$ & $\begin{array}{l}\text { GMFCS } \\
\text { GMFM-88 domains } \\
\text { MACs } \\
\text { Motor type/distribution }\end{array}$ & $\begin{array}{l}\text { Prevalence, } \chi^{2} \\
\text { Binomial logistic regression }\end{array}$ \\
\hline $\mathrm{H} 2(\mathrm{a})$ & $\begin{array}{l}\text { SOMA (overall) Dichotomous } \\
\text { DDS (overall) Dichotomous } \\
\text { PSAS (overall) Dichotomous } \\
\text { Pharyngeal signs (overall) Dichotomous } \\
\text { Saliva control (overall) Dichotomous }\end{array}$ & $\begin{array}{l}\text { GMFCS } \\
\text { GMFM-88 domains } \\
\text { MACs } \\
\text { Motor type/distribution }\end{array}$ & $\begin{array}{l}\text { Prevalence, } \chi^{2} \\
\text { Binomial logistic regression }\end{array}$ \\
\hline $\mathrm{H} 2(\mathrm{~A})$ & $\begin{array}{l}\text { DDS Part } 2 \text { raw score Continuous } \\
\text { Dysphagia Severity Score Ordinal }\end{array}$ & $\begin{array}{l}\text { GMFM-66 } \\
\text { GMFCS }\end{array}$ & $\begin{array}{l}\text { Linear regression } \\
\text { Multinomial logistic regression }\end{array}$ \\
\hline $\mathrm{H} 2(\mathrm{~B})$ & $\begin{array}{l}\text { Growth (height/length, knee and upper } \\
\text { arm length) Ax1 Continuous }\end{array}$ & OPD and subtypes Ax1 & Linear regression \\
\hline $\mathrm{H} 2(\mathrm{~B})$ & $\begin{array}{l}\text { Nutritional Status (skin-folds, BMI) Ax1 } \\
\text { Continuous }\end{array}$ & OPD and subtypes Ax1 & Linear regression \\
\hline $\mathrm{H} 3$ & $\begin{array}{l}\text { OPD, SOMA, DDS, Pharyngeal Signs, } \\
\text { Saliva Control, Parent Report Ax2 } \\
\text { Dichotomous }\end{array}$ & $\begin{array}{l}\text { OPD, SOMA, DDS, PSAS, } \\
\text { Pharyngeal Signs, Saliva Control, } \\
\text { Parent Report Ax1 Dichotomous }\end{array}$ & $\begin{array}{l}\chi^{2} \text { to compare prevalence } \\
\text { Binomial logistic regression }\end{array}$ \\
\hline H3 & OPD at Ax1 Dichotomous & GMFCS (collapsed) & Binomial logistic regression \\
\hline H3 & OPD at Ax2 Dichotomous & GMFCS (collapsed) & Binomial logistic regression \\
\hline H3 & $\begin{array}{l}\text { Nutritional Interventions (tube feeding } \\
\text { and/ or supplements) Ax2 Ordinal }\end{array}$ & OPD and subtypes Ax1 & Multinomial logistic regression \\
\hline H3 & $\begin{array}{l}\text { Growth (height/length, knee and upper } \\
\text { arm length) Ax2 Continuous }\end{array}$ & OPD and subtypes $A x 1$ & Linear regression \\
\hline $\mathrm{H} 3$ & $\begin{array}{l}\text { Nutritional Status (skin-folds, BMI) Ax2 } \\
\text { Continuous }\end{array}$ & OPD and subtypes Ax1 & Linear regression \\
\hline
\end{tabular}

children in GMFCS $\mathrm{V}$ have been reported to have OPD. ${ }^{14}$ With an expected 40 participants per GMFCS level (total $\mathrm{n}=200$ ), this study will be able to detect a significant difference between groups ( $80 \%$ power, $\alpha=0.05$ ) if the true proportion of OPD in the population differs by $>25 \%$ between groups.

\section{Validity and reproducibility studies \\ Oropharyngeal dysphagia reproducibility study}

With an expected agreement of greater than $90 \%$, a sample of 20 children with CP per age band (a total of 40 children across 18-36 months age range and gross motor severity levels) will be able to give sufficient statistical power, with $95 \%$ confidence.

\section{Oropharyngeal dysphagia discriminative validity study}

In order to estimate the true mean score of typically developing children aged 18-24 and 30-36 months on the SOMA and DDS with 95\% confidence (and precision of 0.5 around the estimate), a reference sample of 16 typically developing children from each age band (ie, $\mathrm{n}=16$ 18-24 months corrected age; $\mathrm{n}=16$ 30-36 months corrected age) will be needed. In total, we propose to recruit 40 children aged 18-36 months.

An estimate of the standard deviation of 0.3 for the typically developing group was based on a previous sample of typically developing children participating in the GNPA study aged 4 years (scored on the DDS). It is expected that the variability in the younger age range will be greater than the 4-year-old sample, and therefore a standard deviation of 0.5 was used to ensure that the sample is large enough to give precision to the estimate of mean scores. The DDS is the measure expected to have the greatest variability in scores, and therefore it has been used for the sample size calculations.

\section{Statistical considerations}

This study explores the relationship between OPD as an outcome variable (overall, impairment in saliva control, oral and pharyngeal phases and food/fluid textures) with the primary exposure variable of gross motor skill attainment. It also investigates OPD as an exposure variable for the outcomes of growth and nutritional status. The statistical analysis plan is summarised in table 2 . Demographic data of the sample will be presented with descriptive statistics, and sample representativeness to the population determined by comparing the prevalence of GMFCS classifications to the non-participants and data reported in an Australian register study. ${ }^{68}$

Inter-rater and intrarater reliability of the primary measures (SOMA, DDS, PSAS, pharyngeal signs, saliva control and GMFCS) will be assessed using Cohen's Kappas 
(weighted and unweighted), and percentage agreement will be used. Existing cut scores for the SOMA, DDS and PSAS will be evaluated for their sensitivity and specificity to accurately identify typically developing children as having no oropharyngeal dysphagia. The mean score of the typically developing reference sample $\left(\mathrm{Mean}_{\mathrm{TDC}}\right)+\mathrm{two}$ $\mathrm{SD}$ will be used to determine more appropriate cut scores for the measures (ie, scores above two SD of the Mean ${ }_{\text {TDC }}$ are considered to indicate the presence of oropharyngeal dysphagia). The reference sample will be included in regression analyses for the overall study as a base group for comparison.

The strength of relationship between outcome and exposure variables will be analysed using regression modelling with ORs (for binary outcome variables) and relative risk ratios (for ordinal outcome variables). The 95\% CIs will be calculated for all effect estimates. GMFCS levels will be collapsed into three groups (GMFCS I-II, GMFCS III and GMFCS IV-V) for regression models in the longitudinal study $(\mathrm{n}=60)$ to increase statistical power. All demographic data, such as age, gender and geographical location, will be used in regression models to explore potential confounding with the primary variables. Postcode will be used to allocate children into five geographical categories from highly accessible to very remote. ${ }^{69}$ Likelihood ratios will be used to evaluate the influence of covariates on the models, using backward stepwise elimination. If a group within a model has perfect prediction of the outcome, ORs will be calculated after applying a continuity correction of 0.5 to each appropriate cell. All data analyses will be performed using Stata Statistical Software. ${ }^{70}$ For all tests, significance will be set at $\mathrm{p}<0.05$.

\section{ETHICS AND DISSEMINATION}

Ethics committee approvals have been gained through the University of Queensland Medical Research Ethics Committee (2008002260), the Children's Health Services District Ethics Committee (HREC/08/QRCH/112), the Mater Health Services Human Research Ethics Committee (1520EC), the Cerebral Palsy League of Queensland (CPLQ 2008/ 2010 1029), Gold Coast Health Service District Human Research Ethics Committee (HREC/09/ QGC/88), Central Queensland Health Services District Human Research Ethics Committee (SSA/10/QCQ/13) and the Townsville Health Service District Human Research Ethics Committee (HREC/09/QTHS/96). There are no known health or safety risks associated with participation in any aspect of the described study. All families will give written informed consent to participate, and they are able to withdraw their child from the study at any time without explanation, without any penalty from staff at the Royal Children's Hospital or University of Queensland, or any effect on their child's care. Data collected in this study will be stored in a coded reidentifiable form (by ID number). Each child has three assessment appointments across the duration of the larger study, which necessitates data to be reidentifiable.

To our knowledge, this protocol outlines the first large population-based study using direct clinical feeding assessment in young children with CP. The results of this study are planned to be published in peer reviewed medical and clinical journals, and presented at relevant international conferences. The following publications are proposed:

- Validity and reproducibility of measures of orophayrngeal dysphagia for young children with CP.

- Oropharyngeal dysphagia in young children with CP and its relationship to gross motor skills.

- Oral phase impairment in young children with CP.

- Pharyngeal phase impairment in young children with C.P.

- Functional feeding skills, food and fluid texture inclusion in diets of young children with CP.

- Longitudinal relationships between orophayrngeal dysphagia, gross motor skills, growth and nutritional status in young children with CP.

\section{Author affiliations}

${ }^{1}$ Queensland Cerebral Palsy and Rehabilitation Research Centre, Discipline of Paediatrics and Child Health, School of Medicine, The University of Queensland, Brisbane, Australia

${ }^{2}$ Queensland Children's Medical Research Institute, The University of Queensland, Brisbane, Australia

${ }^{3}$ Department of Speech Pathology, Royal Children's Hospital, Brisbane, Australia

${ }^{4}$ Children's Nutrition Research Centre, Discipline of Paediatrics and Child Health, School of Medicine, The University of Queensland, Brisbane, Australia ${ }^{5}$ School of Population Health, The University of Queensland, Brisbane, Australia

Contributions KAB, KAW, KLB and RNB contributed to the study protocol. PSWD, RNB, KLB, RSW and KAW contributed to study concept, design and grant writing. All authors read, critically revised and approved the final manuscript.

Funding This work was supported by the National Health and Medical Research Council (NHMRC) Postgraduate Medical and Dental Scholarship (1018264-KAB), NHMRC Career Development Fellowship (APP1037220RNB) and NHMRC Research Grants (569605 and 465128).

\section{Competing interests None.}

Ethics approval University of Queensland Medical Research Ethics Committee (2008002260).

Provenance and peer review Not commissioned; externally peer reviewed.

Data sharing statement Further details of the study protocol can be requested from the corresponding author.

\section{REFERENCES}

1. Stevenson RD, Haves RP, Cater LV, et al. Clinical correlates of linear growth in children with cerebral palsy. Dev Med Child Neurol 1994;36:135-42.

2. Krick J, Van Duyn MS. The relationship between oral-motor involvement and growth: a pilot study in a pediatric population with cerebral palsy. J Am Diet Assoc 1984;84:555-9.

3. Cerebral Palsy Institute. Australian Cerebral Palsy Register Report: Birth years 1993-2003. 2009. https://secure.cpregister-aus.com.au/ pubs/pdf/ACPR-Report.pdf (accessed 24 Apr 2012).

4. Blair E, Badawi N, Watson L. Definition and classification of the cerebral palsies: the Australian view. Dev Med Child Neurol 2007;49:33. 
5. Waterman ET, Koltai PJ, Downey JC, et al. Swallowing disorders in a population of children with cerebral palsy. Int $J$ Pediatr Otorhinolaryngol 1992;24:63-71.

6. Matsuo K, Palmer JB. Anatomy and physiology of feeding and swallowing -normal and abnormal. Phys Med Rehabil Clin N Am 2008:19:691-707.

7. Brodsky L, Arvedson JC. Anatomy, embryology, physiology, and normal development. In: Arvedson JC, Brodsky L. eds. Pediatric swallowing and feeding: assessment and management. 2nd edn. Canada: Thomson Delmar Learning, 2002:3-13.

8. Morgan A, Reilly A. Clinical signs, aeitiologies and characteristics of paediatric dysphagia. In: Cichero J, Murdoch BE. eds. Dysphagia: foundation, theory and practice. Chichester, England: John Wiley \& Sons, 2006:391-465.

9. Rogers B, Arvedson JC, Buck G, et al. Characteristics of dysphagia in children with cerebral palsy. Dysphagia 1994;9:69-73.

10. DeMatteo C, Matovich D, Hjartarson A. Comparison of clinical and videofluoroscopic evaluation of children with feeding and swallowing difficulties. Dev Med Child Neurol 2005;47:149-57.

11. Rossi T, Brodsky L, Arvedson JC. Pediatric gastroenterology. In: Arvedson JC, Brodsky L. eds. Pediatric swallowing and feeding: assessment and management. 2nd edn. Canada: Thomson Delmar Learning, 2002:187-231.

12. Stallings VA, Charney EB, Davies JC, et al. Nutrition-related growth failure of children with quadraplegic cerebral palsy. Dev Med Child Neurol 1993;35:126-38.

13. Wilson EM, Hustad KC. Early feeding abilities in children with cerebral palsy: a parental report study. J Med Speech Lang Pathol 2009;17:31-44.

14. Calis EA, Veugelers R, Sheppard JJ, et al. Dysphagia in children with severe generalized cerebral palsy and intellectual disability. Dev Med Child Neurol 2008;50:625-30.

15. Fung EB, Samson-Fang L, Stallings VA, et al. Feeding dysfunction is associated with poor growth and health status in children with cerebral palsy. J Am Diet Assoc 2002;102:361-73.

16. Sullivan PB, Lambert B, Rose M, et al. Prevalence and severity of feeding and nutritional problems in children with neurological impairment: Oxford Feeding Study. Dev Med Child Neurol 2000;42:674-80.

17. Dahl M, Thommessen M, Rasmussen M, et al. Feeding and nutritional characteristics in children with moderate or severe cerebral palsy. Acta Paediatr 1996;85:697-701.

18. Ortega ADOL, Ciamponi AL, Mendes FM, et al. Assessment scale of the oral motor performance of children and adolescents with neurological damages. J Oral Rehabil 2009;36:653-9.

19. Field D, Garland M, Williams K. Correlates of specific childhood feeding problems. J Paediatr Child Health 2003;39:299-304.

20. Reilly S, Skuse D, Poblete X. Prevalence of feeding problems and oral motor dysfunction in children with cerebral palsy: a community survey. J Pediatr 1996;129:877-82.

21. Thommessen M, Heiberg A, Kase BF, et al. Feeding problems, height and weight in different groups of disabled children. Acta Paediatr Scand 1991;80:527-33

22. Love RJ, Hagerman EL, Taimi EG. Speech performance, dysphagia and oral reflexes in cerebral palsy. J Speech Hear Disord 1980; XLV:59-75

23. Erkin G, Culha C, Ozel S, et al. Feeding and gastrointestinal problems in children with cerebral palsy. Int $J$ Rehabil Res 2010;33:218-24.

24. Parkes J, Hill NAN, Platt MJ, et al. Oromotor dysfunction and communication impairments in children with cerebral palsy: a register study. Dev Med Child Neurol 2010;52:1113-19.

25. Santoro A, Dasso Lang MB, Moretti E, et al. A proposed multidisciplinary approach for identifying feeding abnormalities in children with cerebral palsy. J Child Neurol 2012;27:708-12

26. Yilmaz S, Basar P, Gisel EG. Assessment of feeding performance in patients with cerebral palsy. Int J Rehabil Res 2004;27:325-9.

27. Bosma JF. Development of feeding. Clin Nutr 1986;5:210-18.

28. Cichero J. Swallowing from infancy to old age. In: Cichero J, Murdoch BE. eds. Dysphagia: foundation, theory and practice. Chichester, England: John Wiley \& Sons, 2006:27-46.

29. Arvedson JC, Brodsky L, Reigstad D. Clinical feeding and swallowing assessment. In: Arvedson JC, Brodsky L. eds. Pediatric swallowing and feeding. 2nd edn. Canada: Thomson Delmar Learning, 2002:283-340.

30. Lanert G, Ekberg O. Positioning improves the oral and pharyngeal swallowing function in children with cerebral palsy. Acta Paediatr 1995;84:689-92.
31. Kelly NB, Huckabee ML, Jones RD, et al. Integrating swallowing and respiration: preliminary results of the effect of body position. $J$ Med Speech Lang Pathol 2007;15:347-55.

32. Rogers B. Feeding method and health outcomes of children with cerebral palsy. J Pediatr 2004;145:S28-32.

33. Eyman RK, Grossman HJ, Chaney RH. Thel life expectancy of profoundly handicapped people with mental retardation. $N$ Engl $J$ Med 1990;323:584-9.

34. Strauss D, Ashwal S, Shavelle R. Prognosis for survival and improvement in function in children with severe developmental disabilities. J Pediatr 1997;131:712-17.

35. Parkes J, Hill N. The needs of children and young people with cerebral palsy. Paediatr Nurs 2010;22:14-19.

36. Patrick J, Boland M, Stoski D, et al. Rapid correction of wasting in children with cerebral palsy. Dev Med Child Neurol 1986; 28:734-9.

37. Mirrett PL, Riski JE, Glascott J, et al. Videofluoroscopic assessment of dysphagia in children with severe spastic cerebral palsy. Dysphagia 1994:9:174-9.

38. Bell KL, Boyd RN, Tweedy SM, et al. A prospective, longitudinal study of growth, nutrition and sedentary behaviour in young children with cerebral palsy. BMC Public Health 2010;10:179.

39. Boyd R, Ada L. Physiotherapy management of spasticity. In: Barnes MPJohnson GR. eds. Upper motor neurone syndrome and spasticity clinical management and neurophysiology. 2nd edn. Cambridge, NY: Cambridge University Press, 2008:79-98.

40. Russell DJ, Rosenbaum PL, Avery LM, et al. Gross motor function measure (GMFM-66 and GMFM-88) user's manual. London: Mac Keith, 2002.

41. Eliasson AC, Krumlinde Sundholm L, Rosblad B, et al. The Manual Ability Classification System (MACS) for children with cerebral palsy: scale development and evidence of validity and reliability. Dev Med Child Neurol 2006;48:549-54.

42. Haley SM, Coster WJ, Ludlow LH, et al., Pediatric Evaluation of Disability Inventory (PEDI). Development, standardization and administration manual. Boston, MA: PEDI Research Group, 1998.

43. Reilly S, Skuse D, Wolke D. Schedule for oral motor assessment: administration manual. London: Whurr Publishers Ltd, 2000.

44. Morris SE. A longitudinal study of feeding and pre-speech skills from birth to three years. [Unpublished research study], 2003.

45. Lefton-Greif MA, McGrath-Morrow SA. Deglutition and respiration: development, coordination, and practical implications. Semin Speech Lang 2007;28:166-79.

46. Morris SE. Pre-Speech Assessment Scale: a rating scale for the measurement of pre-speech behaviors from birth through two years. Clifton, NJ: J.A. Preston Corp, 1982

47. Benfer KA, Weir KA, Boyd RN. Clinimetrics of measures of oropharyngeal dysphagia for preschool children with cerebral palsy and neurodevelopmental disabilities: a systematic review. Dev Med Child Neurol Published Online First: 14 May 2012. doi: 10.1111/j. 1469-8749.2012.04302.x

48. Sheppard JJ. Dysphagia disorders survey and dysphagia management staging scale (adult and pediatric applications): user's manual: Australian edition. Ryde, NSW: The Centre for Developmental Disability, 2003

49. Skuse D, Stevenson J, Reilly S, et al. Schedule for oral-motor assessment (SOMA): Methods of validation. Dysphagia 1995; 10:192-202.

50. Reilly S, Skuse D, Mathisen B, et al. The objective rating of oral-motor functions during feeding. Dysphagia 1995;10: 177-91.

51. Sheppard JJ, Liou J, Hochman R, et al. Nutritional correlates of dysphagia in individuals institutionalized with mental retardation. Dysphagia 1988;3:85-9.

52. Weir K, McMahon S, Barry L, et al. Clinical signs and symptoms of oropharyngeal aspiration and dysphagia in children. Eur Respir $J$ 2009;33:604-11.

53. Thomas-Stonell N, Greenberg J. Three treatment approaches and clinical factors in the reduction of drooling. Dysphagia 1988;3:73-8.

54. Senner JE, Logemann J, Zecker S, et al. Drooling, saliva production, and swallowing in cerebral palsy. Dev Med Child Neurol 2004;46:801-6.

55. Palisano R, Rosenbaum P, Walter RS, et al. Development and reliability of a system to classify gross motor function in children with cerebral palsy. Dev Med Child Neurol 1997;39:214-23.

56. Wood EC, Rosenbaum P. The Gross Motor Function Classification System for cerebral palsy: a study of reliability and stability over time. Dev Med Child Neurol 2000;42:292-6. 
57. Oeffinger D, Tylkowski C, Rayens M, et al. Gross Motor Function Classification System and outcome tools for assessing ambulatory cerebral palsy: a multicenter study. Dev Med Child Neurol 2004:46:311-19.

58. Gorter J, Ketelaar M, Rosenbaum P, et al. Use of the GMFCS in infants with CP: the need for reclassification at age 2 years or older. Dev Med Child Neurol 2009;51:46-52.

59. Gorter J, Rosenbaum P, Palisano R, et al. Limb distribution, motor impairment and functional classification of cerebral palsy. Dev Med Child Neurol 2004;46:461-7.

60. Stevenson RD. Use of segmental measures to estimate stature in children with cerebral palsy. Arch Pediatr Adolesc Med 1995;149:658-62.

61. Addo OY, Himes JH. Reference curves for triceps and subscapular skinfold thicknesses in US children and adolescents. Am J Clin Nutr 2010;91:635-42.

62. Walker JL, Bell KL, Caristo FM, et al. A review of energy intake measures used in young children with cerebral palsy. Dev Med Child Neurol 2011;53:569.

63. Atherton M, Bellis-Smith N, Cichero J, et al. Texture-modified foods and thickened fluids as used for individuals with dysphagia: Australian standardised labels and definitions. Nutr Diet 2007;64: s53-76.
64. Russell DJ, Rosenbaum PL, Cadman DT, et al. The gross motor function measure: a means to evaluate the effects of physical therapy. Dev Med Child Neurol 1989;31:341-52.

65. Russell DJ, Avery LM. Improved scaling of the gross motor function measure for children with cerebral palsy: evidence of reliability and validity. Phys Ther 2000;80:873-85.

66. Sanger TD, Delgado MR, Gaebler-Spira D, et al. Classification and definition of disorders causing hypertonia in childhood. Pediatrics 2003;111:e89-98.

67. Plasschaert VFP, Ketelaar M, Nijnuis MG, et al. Classification of manual abilities in children with cerebral palsy under 5 years of age: how reliable is the Manual Ability Classification System? Clin Rehabil 2009;23:164-70.

68. Howard J, Soo B, Graham H, et al. Cerebral palsy in Victoria: motor types, topography and gross motor function. J Paediatr Child Health 2005;41:479-83.

69. Commonwealth Department of Health and Aged Care. Measuring remoteness: Accessibility/Remoteness Index of Australia (ARIA). 2001. http://www.health.gov.au/internet/main/publishing.nsf/Content/ 7B1A5FA525DD0D39CA25748200048131/\$File/ocpanew14.pdf (accessed 8 Mar 2012).

70. Statacorp. Statistical Software: Release 10.0. College Station, TX: Stata Corporation, 2007. 


\section{Correction}

Benfer KA, Weir KA, Bell KL, et al. Longitudinal cohort protocol study of oropharyngeal dysphagia: relationships to gross motor attainment, growth and nutritional status in preschool children with cerebral palsy. BMJ Open 2012;2:e001460. A number of author corrections were inadvertently missed during the proofing stage:

1. The title of this paper should read: 'Protocol for a longitudinal cohort study of oropharyngeal dysphagia: relationships to gross motor attainment, growth and nutritional status in preschool children with cerebral palsy.'

2. Under the section "Aims and hypotheses" the expansion of DDS is actually "Dysphagia Disorders Survey" (not "Schedule").

3. Under the section "Thomas-Stonell \& Greenburg Scale-saliva control", paragraph 2, reference 53 should be after "Scale" (as this is part of the measure name).

4. Table 2: H2(A) -These are all the same hypothesis, so all instances should have been in upper case A.

We apologise for these errors.

BMJ Open 2012;2:e001460corr1. doi:10.1136/bmjopen-2012-001460corr1 